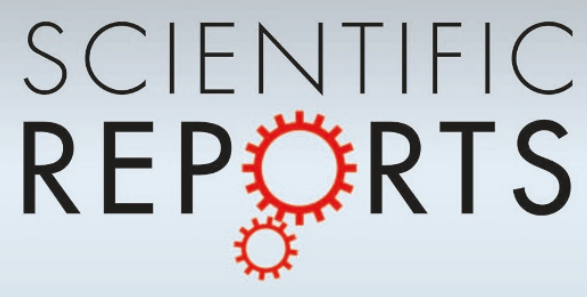

OPEN

SUBJECT AREAS:

OPTICAL PROPERTIES AND

DEVICES

OPTOELECTRONIC DEVICES AND

COMPONENTS

Received

23 December 2013

Accepted

11 June 2014

Published

27 June 2014

Correspondence and requests for materials should be addressed to

H.Z. (HZhang@szu. edu.cn); S.C.W.

(SCWen@hnu.edu.cn) or D.Y.T. (EDYTang@ ntu.edu.sg)

* These authors contributed equally to this work.

\section{Critical coupling with graphene-based hyperbolic metamaterials}

\author{
Yuanjiang Xiang ${ }^{1,2 *}$, Xiaoyu Dai ${ }^{2 *}$, Jun Guo ${ }^{1,3}$, Han Zhang ${ }^{2}$, Shuangchun Wen ${ }^{3} \&$ Dingyuan Tang $^{1}$
}

\begin{abstract}
${ }^{1}$ School of Electrical and Electronic Engineering, Nanyang Technological University, Singapore 639798, ${ }^{2}$ SZU-NUS Collaborative Innovation Centre for Optoelectronic Science \& Technology, College of Optoelectronic Engineering, Shenzhen University, Shenzhen 518060, China, ${ }^{3}$ Key Laboratory for Micro-/Nano-Optoelectronic Devices of Ministry of Education, College of Physics and Microelectronic Science, Hunan University, Changsha 410082, China.
\end{abstract}

In order to effectively realize and control the critical coupling, a graphene-based hyperbolic metamaterial has been proposed to replace the absorbing thin film in the critically coupled resonance structure. Our calculations demonstrate that the critical coupling effect (near-perfect light absorption) can be achieved at the near-infrared wavelength by using this layered structure, while the critical coupling frequency can be tuned by varying the Fermi energy level of graphene sheets via electrostatic biasing. Moreover, we show that the critical coupling frequency can be tuned by changing the thickness of the dielectric or layer number of the graphene sheets in the unit cell of the graphene-dielectric HMM. The optimization performance has also been indicated, which may offer an opportunity towards the experimental designs of high efficient graphene based critical coupling devices.

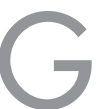
raphene has attracted intense scientific interests owing to its outstanding optical properties including the strong light-graphene interaction, the broadband and high-speed operation, giant nonlinear Kerr index (several orders of magnituede larger than conventional bulk media) and the controllable optical properties due to tunability of the charge carrier density by applying the bias voltage upon graphene ${ }^{1-8}$. Graphene also exhibits wavelength insensitive absorption in the visible- and near-infrared frequencies, which makes graphene a potential candidate as a building block for optical detectors and photovoltaic devices. However, absorption of the single-layer graphene remains very weak. Some methods had been proposed to enhance its absorption. Thongrattanasiri et al. demonstrated that $100 \%$ light absorption could occur if a single patterned sheet of doped graphene is designed instead ${ }^{9}$, Ferreira et al. reported that the absorption of graphene can reach up to $100 \%$ within Fabry-Pérot cavity sandwiched graphene structures ${ }^{10}$ or in graphene-metamaterial corrugated structures ${ }^{11}$, Nikitin et al. showed that graphene periodic antidot arrays could provide a strong electromagnetic absorption at both microwave and $\mathrm{THz}$ regions ${ }^{12}$, Nefedov et al. demonstrated that $100 \%$ light absorption could also be achieved in a graphene-based asymmetric hyperbolic metamaterial $(\mathrm{HMM})^{13}$. In this paper, we show that near-perfect light absorption can be obtained by the critical coupling within graphene-based HMMs.

A critically coupled resonator (CCR) is a thin-film structure that can absorb nearly all of the incident electromagnetic radiation, leading to the null scattering. It was firstly reported by Tischler ${ }^{14,15}$, who experimentally demonstrated that a $5 \mathrm{~nm}$ thick film of high oscillator strength J-aggregated dye separated from a dielectric Bragg reflector by a dielectric spacer can absorb more than $97 \%$ of the incident light at the wavelength $\lambda=591 \mathrm{~nm}^{14}$. In the following, Gupta et al. replaced the polymer absorbing layer by a metaldielectric composite film, and showed that the critical coupling (CC) frequency can be tuned by changing the volume fraction ${ }^{16}$. For a larger volume fraction, CC at two distinct frequencies can be obtained due to the strong coupling. Subsequently they extended their studies to oblique incidence for both the TE and TM polarizations ${ }^{17}$, Fabry-Perot cavity with metamaterial mirrors ${ }^{18}$, or layered media with Kerr nonlinearity ${ }^{19}$. However, a critically coupled system has limited flexibility for a given absorber material. The tunability of CC is intrinsically important for applications in optical devices. It is desired to tune CC in a fixed configuration or device by an external field. For this purpose, introducing graphene sheets into a CCR seems to be a good approach for achieving CC. Here, we replace the polymer absorbing layer or metal-dielectric composite film by the graphene-based $\mathrm{HMMs}^{20-23}$ composed of stacked graphene sheets separated by thin dielectric layers. It is found that such a graphene-based HMM can support controllable CC at a single wavelength. 
(a)

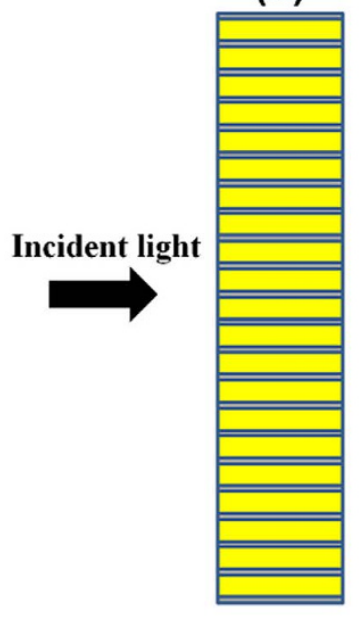

(b)

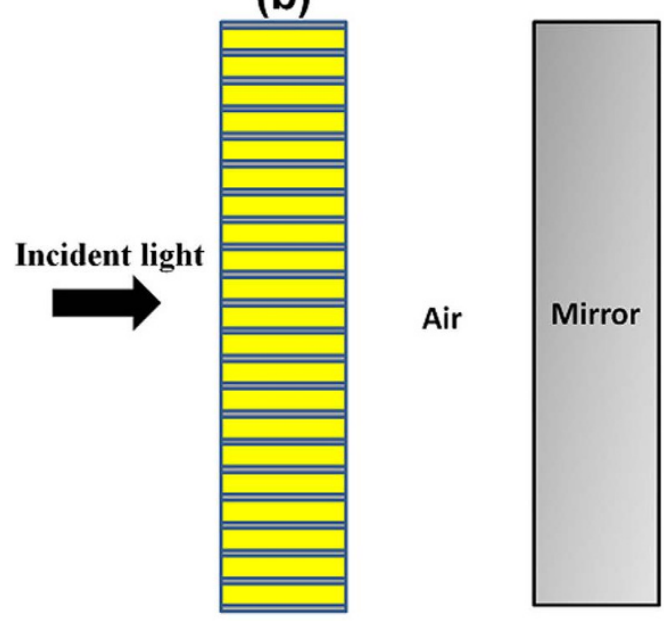

(c)

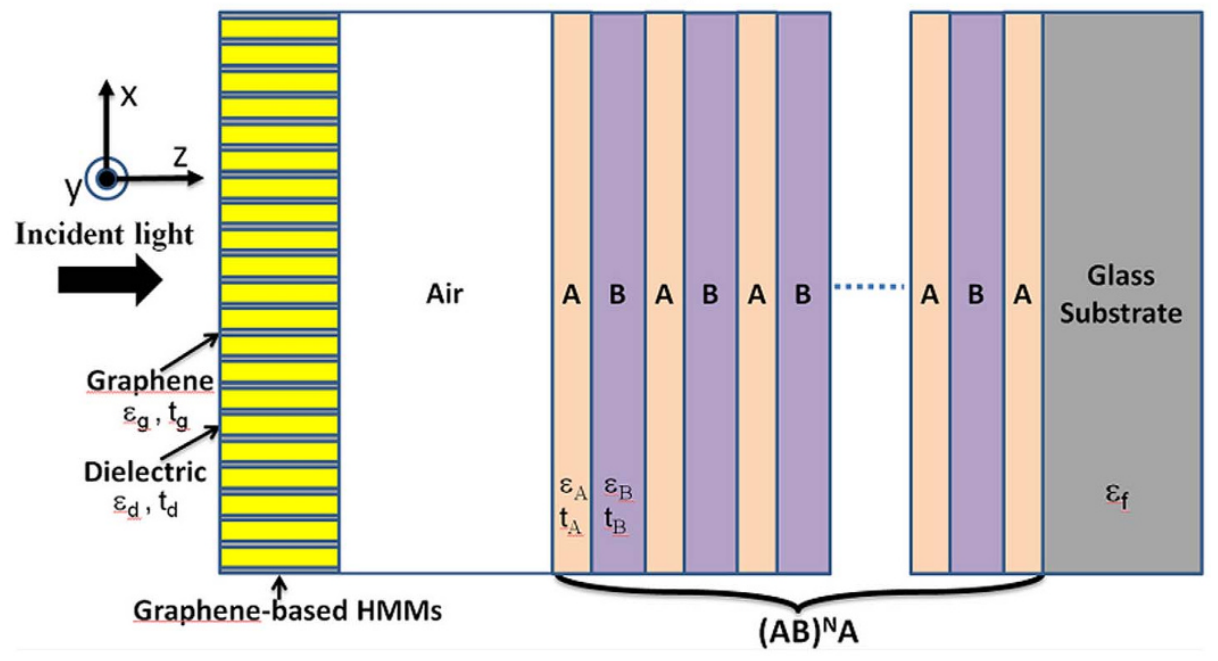

Figure $1 \mid$ (a) Geometry for a graphene-based hyperbolic metamaterial (HMM). (b) A critically coupled resonator with the graphene-based HMMs and perfect mirror spaced by the air. (c) The critically coupled resonator with the graphene-based HMM and the dielectric distributed Bragg reflector.

\section{Results}

Graphene-based hyperbolic metamaterials. Our graphene-based HMM is representatively shown in Fig. 1(a). It is a graphene multilayer structure in which separations between graphene sheets are assumed to be filled with the dielectric. For the HMM in air, as shown in Fig. 1(a), the absorption is smaller than 50\% generally and the perfect absorber cannot be constructed. In order to realize the perfect absorption, we try to construct a CCR by introducing a lossless metallic mirror to the back of the HMMs, as shown in Fig. 1(b). In the experiment, lossless metallic mirrors may be replaced by more realistic dielectric Bragg reflectors (DBRs). The designed CCR has been shown in Fig. 1(c), which is consisted by a graphene-based HMM separated from a DBR by a dielectric spacer. The graphene-based HMM acts as a thin absorbing film, which is created by alternating layers of graphene with the surface conductivity $\sigma$ and slab thickness $t_{\mathrm{g}}$ and conventional dielectric with a dielectric constant $\varepsilon_{\mathrm{d}}$ and slab thickness $t_{\mathrm{d}}$. The period of the unit cell of the layered structure is $t=t_{d}+t_{g}$. The graphene's surface conductivity can be calculated by adopting the Kubo formula ${ }^{24-26}$. Without considering the external magnetic field, the isotropic surface conductivity $\sigma$ of graphene can be written as the sum of the intra-band $\sigma_{\text {intra }}$ and the inter-band term $\sigma_{\text {inter, }}$, where

$$
\begin{gathered}
\sigma_{\text {intra }}=\frac{i e^{2} k_{B} T}{\pi \hbar^{2}(\omega+i / \tau)}\left(\frac{E_{F}}{k_{B} T}+2 \ln \left(e^{-\frac{E_{F}}{k_{B} T}}+1\right)\right), \\
\sigma_{\text {inter }}=\frac{i e^{2}}{4 \pi \hbar} \ln \left|\frac{2 E_{F}-\left(\omega+i \tau^{-1}\right) \hbar}{2 E_{F}+\left(\omega+i \tau^{-1}\right) \hbar}\right|,
\end{gathered}
$$

where $\omega$ is the frequency of the incident light, $E_{\mathrm{F}}$ is the Fermi energy, $\tau$ is the electron-phonon relaxation time, and $T$ is a temperature in $\mathrm{K}$. $\mathrm{e}, \hbar$ and $\mathrm{k}_{\mathrm{B}}$ are the universal constants related to the electron charge, reduced Planck's constant, and Boltzmann constant, respectively. The Fermi energy $E_{F}=\hbar v_{F}\left(\pi n_{2 D}\right)^{1 / 2}$ can be electrically controlled by an applied gate voltage due to the strong dependence of the carrier density $n_{2 \mathrm{D}}$ on the gate voltage, where $v_{\mathrm{F}}=10^{6} \mathrm{~m} / \mathrm{s}$ is the Fermi velocity of electrons. Obviously, $\sigma$ is highly dependent on the work frequency and Fermi energy, which could provide an effective route to achieve an electrically controlled CC phenomenon.

Under the assumption that the electronic band structure of a graphene sheet cannot be affected by the neighboring graphene sheets, graphene's effective permittivity $\varepsilon_{\mathrm{g}}$ can be written as

$$
\varepsilon_{g}=1+\frac{i \sigma}{\varepsilon_{0} \omega t_{g}}
$$



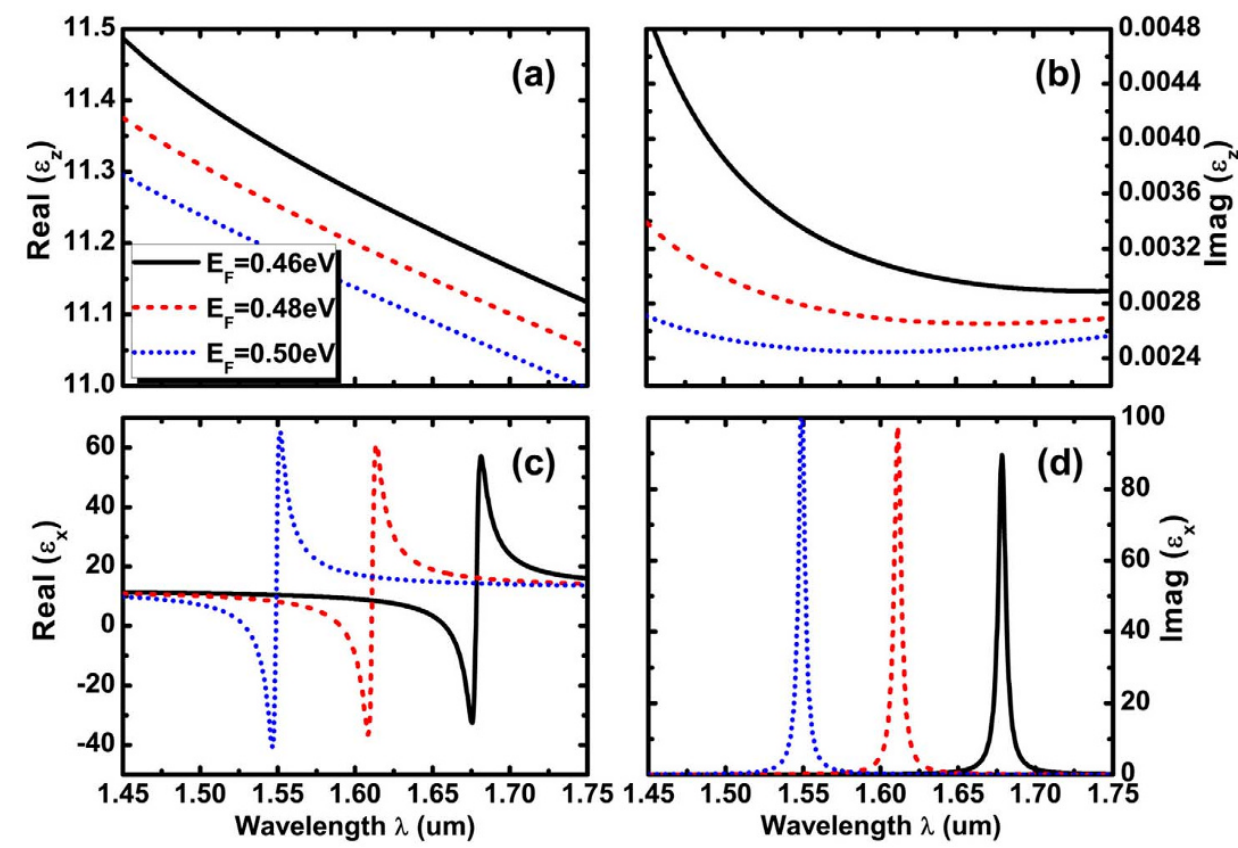

Figure $2 \mid$ (a) and (b) are the real and imaginary parts of $\varepsilon_{\mathrm{z}}$, (c) and (d) are the real and imaginary parts of $\varepsilon_{\mathrm{x}}$. The parameters are $\mathrm{T}=300 \mathrm{~K}, \tau=0.5$ ps, $\mathrm{t}_{\mathrm{g}}$ $=\mathrm{M}^{*} 0.35 \mathrm{~nm}, \mathrm{M}=1, \mathrm{t}_{\mathrm{d}}=8 \mathrm{~nm}, \varepsilon_{\mathrm{d}}=11.7$.

where $\varepsilon_{0}$ is the permittivity in the vacuum. Considering the Cartesian coordinate systems shown in Fig. 1(a), layered structure are illuminated from the left with space-time dependence of fields as $A \exp \left(i k_{z} z\right.$ $\left.+i k_{x} x-i \omega t\right)$. In the sub-wavelength limit, graphene-dielectric layered structure can be treated as a homogeneous effective medium with anisotropic permittivity sensor,

$$
\hat{\varepsilon}=\left\{\begin{array}{ccc}
\varepsilon_{x} & 0 & 0 \\
0 & \varepsilon_{y} & 0 \\
0 & 0 & \varepsilon_{z}
\end{array}\right\},
$$

where $\varepsilon_{x}=\left(f_{g} / \varepsilon_{g}+f_{d} / \varepsilon_{d}\right)^{-1}, \varepsilon_{y}=\varepsilon_{z}=f_{g}{ }^{*} \varepsilon_{g}+f_{d}{ }^{*} \varepsilon_{d}, f_{g}=t_{g} / t$ and $f_{d}=$ $t_{d} / t$ are the filling ratio of graphene sheet and dielectric, respectively. If $\mathrm{t}_{\mathrm{g}} \ll \mathrm{t}_{\mathrm{d}}, \varepsilon_{y}=\varepsilon_{z} \approx \varepsilon_{d}+\frac{i \sigma}{\varepsilon_{0} \omega t_{g}}$, which is consistent with the results by averaging the effective displacement current (including both the displacement current in the dielectric slab and conduction current in the graphene sheet) over the associated electric field in a unit cell ${ }^{20,21}$.

For the TM waves propagating in the graphene-dielectric layered structure, the spatial dispersive curve is given by

$$
\frac{k_{z}^{2}}{\varepsilon_{x}}+\frac{k_{x}^{2}}{\varepsilon_{z}}=k_{0}^{2}
$$

where $\mathrm{k}_{0}$ is the free-space wave vector, $\mathrm{k}_{\mathrm{x}}$ and $\mathrm{k}_{\mathrm{z}}$ are the wave vector along $\mathrm{x}$ and $\mathrm{z}$ direction in the graphene-dielectric layered structure, respectively. If $\varepsilon_{\mathrm{x}} \varepsilon_{\mathrm{z}}<0$, the dispersive curve of Eq. (5) is hyperbolic type, and we call this graphene-dielectric layered structure as HMMs. Since the effective permittivity $\varepsilon_{\mathrm{x}}$ and $\varepsilon_{\mathrm{z}}$ depend on the Fermi energy, the dispersion characteristic of HMM could be tuned by changing the Fermi energy of the graphene sheets.

As an example, in Fig. 2(a)-(d) we plot the effective permittivity $\varepsilon_{\mathrm{x}}$ and $\varepsilon_{\mathrm{z}}$ as a function of the operation wavelength at the different Fermi energies at the near-infrared (NIR) frequency. Here, we assume that the Fermi-energy of graphene $\mathrm{E}_{\mathrm{F}}=0.46,0.48$, and $0.50 \mathrm{eV}$, respectively. The other parameters are $\mathrm{T}=300 \mathrm{~K}, \tau=$ $0.5 \mathrm{ps}, \mathrm{t}_{\mathrm{d}}=8 \mathrm{~nm}, \varepsilon_{\mathrm{d}}=11.7 ; \mathrm{t}_{\mathrm{g}}=\mathrm{M}^{*} 0.35 \mathrm{~nm}$, where $\mathrm{M}$ is the layer number of the graphene sheets, and $\mathrm{M}=1$ is for the single-layer graphene. It is seen from Fig. 2(a) and (b) that the real part of $\varepsilon_{z}$ is positive, and both the real part and the imaginary part of $\varepsilon_{\mathrm{z}}$ vary with wavelength slowly. As the Fermi energy is increased, both the real part and imaginary part of $\varepsilon_{\mathrm{z}}$ decrease slightly. However, $\varepsilon_{\mathrm{x}}$ exhibits resonant behavior in NIR frequency. $\operatorname{Re}\left(\varepsilon_{\mathrm{x}}\right)<0$ and $\operatorname{Im}\left(\varepsilon_{\mathrm{x}}\right)$ become very large near the resonant wavelength $\lambda_{\text {res }}$. For $\mathrm{E}_{\mathrm{F}}=0.50 \mathrm{eV}, \lambda_{\text {res }}$ $=1.534 \mu \mathrm{m}$, at this frequency, $\operatorname{Re}\left(\varepsilon_{\mathrm{x}}\right)<0$ and $\operatorname{Re}\left(\varepsilon_{\mathrm{z}}\right)>0$. Hence Eq. (5) denotes a dispersion curve of hyperboloid. Furthermore, we find that the resonant wavelength of the hyperbolic dispersion curve can be electrically controlled by an applied gate voltage on the graphene sheets. It is clear that the resonant behavior of $\varepsilon_{\mathrm{x}}$ can be tuned by varying the Fermi energy. Increasing Fermi energy, the resonant wavelength $\lambda_{\text {res }}$ shifts to the smaller wavelength; while decreasing Fermi energy results in the red shift of the resonant wavelength $\lambda_{\text {res }}$.

Critical coupling phenomena with graphene-based HMMs. The resonant behavior of $\varepsilon_{\mathrm{x}}$ can be used to design a CCR. We replace the

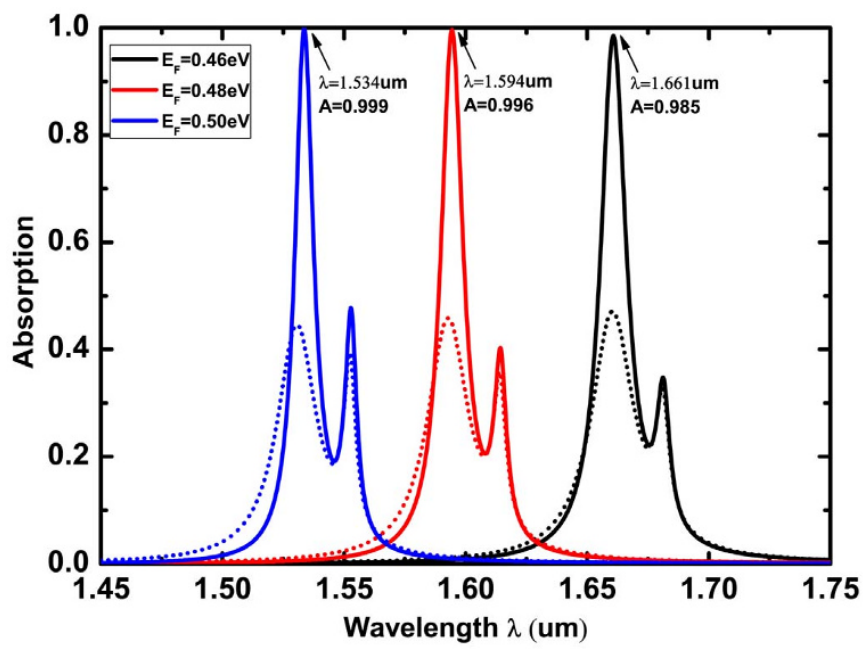

Figure $3 \mid$ The absorption of critically coupled resonance as a function of wavelength at different Fermi energy $E_{F}$, where $t_{s}=600 \mathrm{~nm}, t_{\mathrm{HMM}}=$ $83.5 \mathrm{~nm}$, and the other parameters have the same values as those in Fig. 2(c). The dotted lines are the corresponding absorption of the HMMs on the glass substrate. 

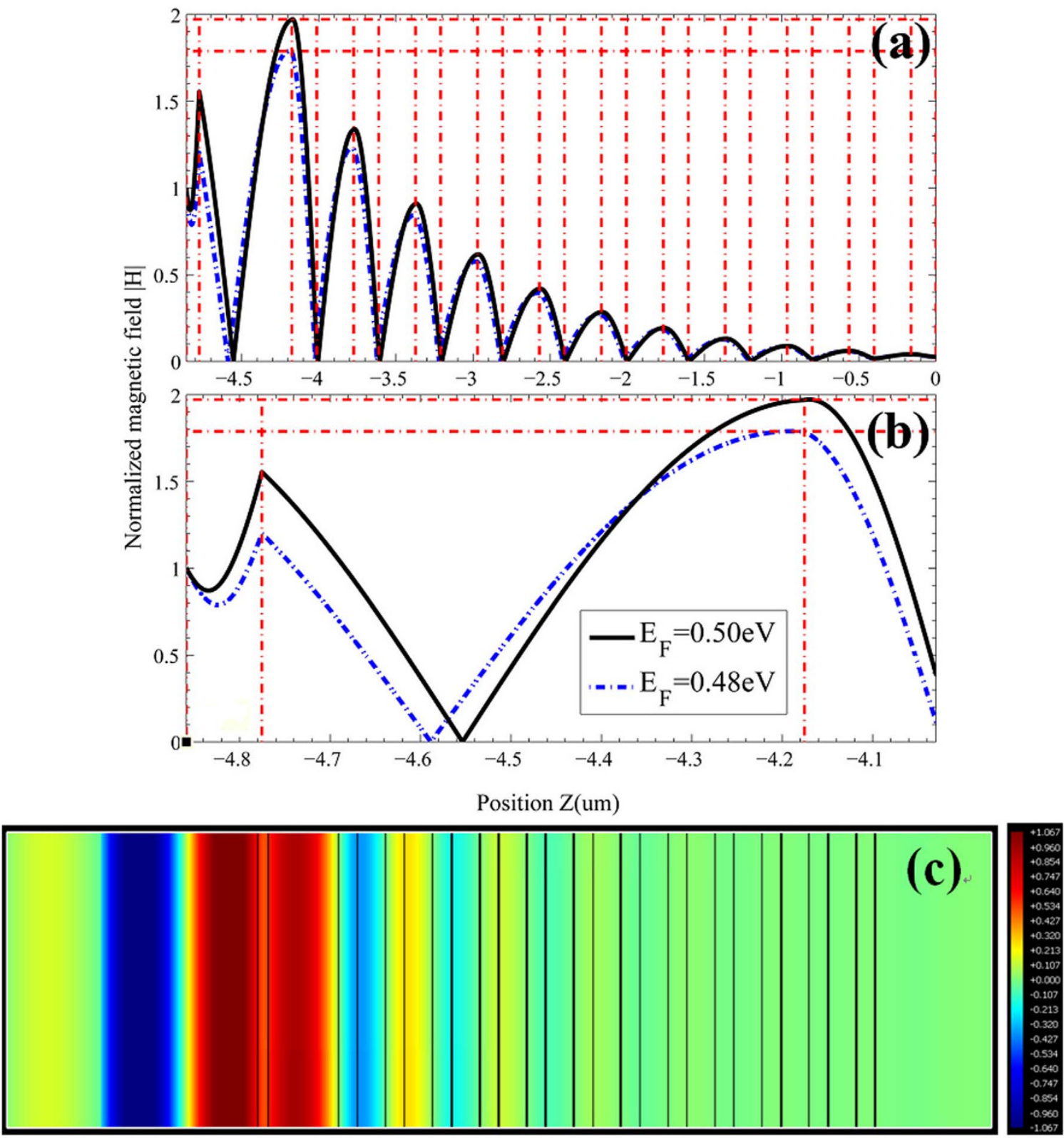

Figure $4 \mid$ The magnetic field distributions in the critically coupled resonance at different Fermi energy $E_{F}$, (b) is the enlarged view of (a) in the graphene-based HMMs and spacer, (c) is the magnetic field distributions gotten by FDTD method for $\mathrm{E}_{\mathrm{F}}=0.50 \mathrm{eV}$ and $\lambda=1.534 \mathrm{um}$. $\mathrm{E}_{\mathrm{F}}=0.50 \mathrm{eV}$ and $\lambda=1.534 \mathrm{um}$, and $\mathrm{E}_{\mathrm{F}}=0.48 \mathrm{eV}$ and $\lambda=1.594 \mathrm{um}$ are for the solid-line and dashed-dotted line, respectively. Other parameters are as in Fig. 2 , the red dashed-dotted lines in (a) and (b) and solid-line in (c) show the interface of different materials.

polymer absorbing layer or the metal-dielectric composite film in the conventional CCRs by the graphene-based HMM, as shown in Fig. 1(a). The DBR $(A B)^{N} A$ is constituted by two dielectrics $A$ and $B$ with permittivity $\varepsilon_{A}$ and $\varepsilon_{B}$ and thickness $t_{A}$ and $t_{B}$ respectively. In the numerical calculations, we choose the following parameters: $\mathrm{N}=$ $10, \varepsilon_{\mathrm{A}}=5.7121$ and $\varepsilon_{\mathrm{B}}=2.6244$. The thickness of dielectrics $\mathrm{A}$ and $\mathrm{B}$ are chosen to correspond to $\lambda / 4$ plate: $t_{A}=\lambda_{c} /\left(4 n_{A}\right)$ and $t_{A}=\lambda_{c} l$ $\left(4 \mathrm{n}_{\mathrm{B}}\right)$, where the central wavelength is chosen as $\lambda_{\mathrm{c}}=1550 \mathrm{~nm}$, $n_{A}=\sqrt{\varepsilon_{A}}$, and $n_{B}=\sqrt{\varepsilon_{B}}$. To easily design and adjust the spatial distance $t_{s}$, we assume that the space between the graphene-based HMM and DBR is filled by air.

Next, we show that CC can be realized in our structure. In Fig. 3 we give the absorption of CCR as a function of wavelength at the different Fermi energy $E_{\mathrm{F}}$. The thickness of spacer layer $\mathrm{t}_{\mathrm{s}}=600 \mathrm{~nm}$ and the thickness of HMM $\mathrm{t}_{\mathrm{HMM}}=10 *\left(\mathrm{t}_{\mathrm{g}}+\mathrm{t}_{\mathrm{d}}\right)=83.5 \mathrm{~nm}$ are chosen to achieve nearly $100 \%$ absorption at the central wavelength $\lambda_{\mathrm{c}}=1.534 \mathrm{um}$ and $\mathrm{E}_{\mathrm{F}}=0.5 \mathrm{eV}$, and the other parameters have the same values as those in Fig. 2. Compared to the absorption $A \approx 40 \%$ of the HMMs on the glass substrate, the absorption from the CCR is enhanced about 2.5 times. Hence, our structure becomes very efficient to maximize light absorption. Another advantage of our structure is the tunable CC frequency by changing the Fermi energy levels, as shown in Fig. 3, which are in consistent with the dependence of the effective permittivity $\varepsilon_{\mathrm{x}}$ on the Fermi energy as shown in Fig. 2(c). When we decrease the Fermi energy $E_{F}$, the absorption peak moves to the longer wavelength side; when we increase the Fermi energy $\mathrm{E}_{\mathrm{F}}$, the absorption peak moves to the longer wavelength side. For $\mathrm{E}_{\mathrm{F}}=0.46 \mathrm{eV}$, the central wavelength is $1.661 \mu \mathrm{m}$; however for $\mathrm{E}_{\mathrm{F}}=0.48 \mathrm{eV}$, the central wavelength $\lambda_{\mathrm{c}}$ is $1.594 \mu \mathrm{m}$. This property suggests that the frequency of absorption peak can be engineered by the Fermi energy of the graphene sheets. Although the absorption remains still high $(>90 \%)$, either the decrease or increase in the Fermi energy level leads to the departure from the CC phenomenon. 


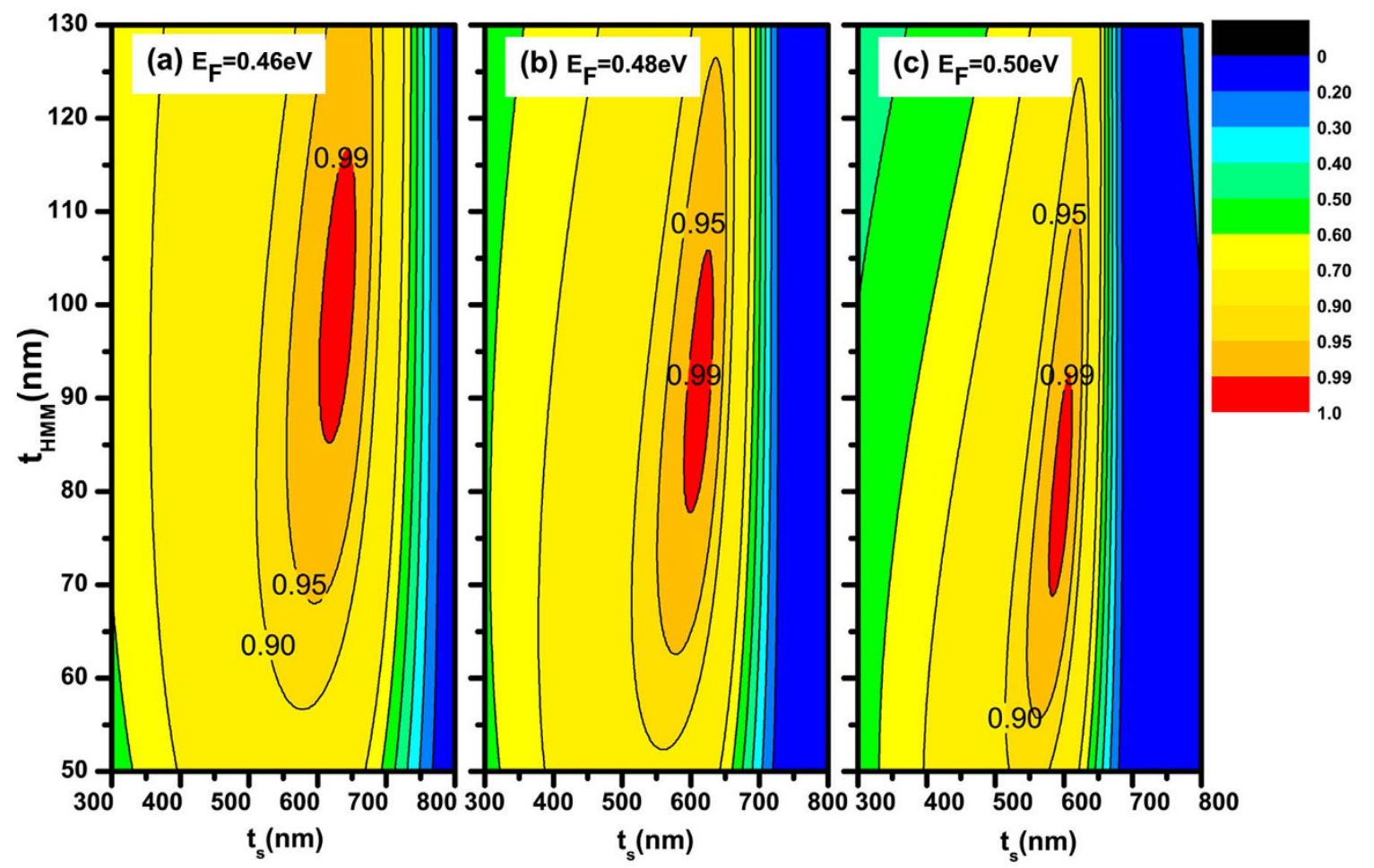

Figure $5 \mid$ The contours of the absorption of critically coupled resonance as a function of thickness of spacer layer $t_{s}$ and thickness of HMMs at the different Fermi energy $\mathrm{E}_{\mathrm{F}}$. Where $\mathrm{E}_{\mathrm{F}}=0.52 \mathrm{eV}$ and $\lambda=1.478 \mathrm{um}$ in $(\mathrm{a}), \mathrm{E}_{\mathrm{F}}=0.50 \mathrm{eV}$ and $\lambda=1.534 \mathrm{um}$ in $(\mathrm{b})$, and $\mathrm{E}_{\mathrm{F}}=0.48 \mathrm{eV}$ and $\lambda=1.594 \mathrm{um}$ in (c). Other parameters are as in Fig. 2.

To understand the almost $100 \%$ absorption of the incident energy by the CCR at the CC frequency, we have plotted the magnetic field distributions inside the layered structure at the CC wavelength $\lambda=$ $1.534 \mathrm{um}$ for $\mathrm{E}_{\mathrm{F}}=0.50 \mathrm{eV}$ based on the transfer matrix method (TMM), as shown in the solid-line in Fig. 4(a) and (b). To confirm the correctness of TMM, in Fig. 4(c) we also provide the simulated magnetic field distributions by using the Finite-difference timedomain (FDTD)(The time evolution of magnetic field has been given in the Supplement materials). The magnetic field amplitude has been normalized to the incident magnetic field amplitude. One can see that the magnetic field is evanescent light wave in DBR due to the total reflection, however the magnetic field is strongly enhanced and localized in the graphene-based HMM and spacer. The large intensity enhancement and large imaginary parts of $\varepsilon_{\mathrm{x}}$ in the absorption layer of graphene-based HMM, explains the CC phenomenon (nearperfect light absorption) at the CC wavelength $\lambda_{\mathrm{c}}=1.534$ um for $\mathrm{E}_{\mathrm{F}}$ $=0.50 \mathrm{eV}$. Additionally, for comparison, in Fig. 4(a) and (b) we have plotted the normalized magnetic field distributions for $\lambda=1.594 \mathrm{um}$ at $\mathrm{E}_{\mathrm{F}}=0.48 \mathrm{eV}$. It is clear that those magnetic fields are weaker than the field at $\mathrm{E}_{\mathrm{F}}=0.50 \mathrm{eV}$, explaining why there is a departure from the $\mathrm{CC}$ phenomenon at $\mathrm{E}_{\mathrm{F}}=0.48 \mathrm{eV}$. Hence, in order to achieve the $\mathrm{CC}$ at the different Fermi energy levels, we must optimize the physical parameters, such as, the fill factors of the dielectric and graphene sheet in the HMMs, the thickness of spacer layer $t_{s}$, and the thickness of HMMs $t_{\mathrm{HMM}}$. However, to realize the tunability of the CC frequency by changing the Fermi energy in a fixed configuration, we intend to finely adjust the distance between the graphene-based HMM and DBR on glass substrate, i.e. the thickness of spacer layer $t_{s}$. The optimization is given as following.

Recover the CC at the different Fermi energy levels. To recover the $\mathrm{CC}$ at the different Fermi energy, we have plotted the contours of the absorption of the CCR as a function of the thickness of the spacer layer $t_{s}$ and the thickness of the HMMs at the different Fermi energy $\mathrm{E}_{\mathrm{F}}$, as shown in Fig. 5. The red region gives the absorption larger than
$99 \%$, here we consider it as the CC region. It is obvious that both $t_{s}$ and $t_{\text {HMM }}$ should be decreased with the increase of Fermi energy. To easily adjust this property in the experiments, we intend to find the common area of the thickness $\mathrm{t}_{\mathrm{HMM}}$ for the $\mathrm{CC}$ at the different Fermi energies. It is found that this region is located near $\mathrm{t}_{\mathrm{HMM}}=88 \mathrm{~nm}$. The optimized results have been demonstrated in Fig. 6. Here we keep the thickness of HMM $t_{\mathrm{HMM}}=88 \mathrm{~nm}$ fixed, and adjust the distance $t_{s}$. According to the optimized results in Fig. 5, we choose $t_{s}$ $=590 \mathrm{~nm}$ for $\mathrm{E}_{\mathrm{F}}=0.50 \mathrm{eV}, \mathrm{t}_{\mathrm{s}}=610 \mathrm{~nm}$ for $\mathrm{E}_{\mathrm{F}}=0.48 \mathrm{eV}$, and $\mathrm{t}_{\mathrm{s}}=$

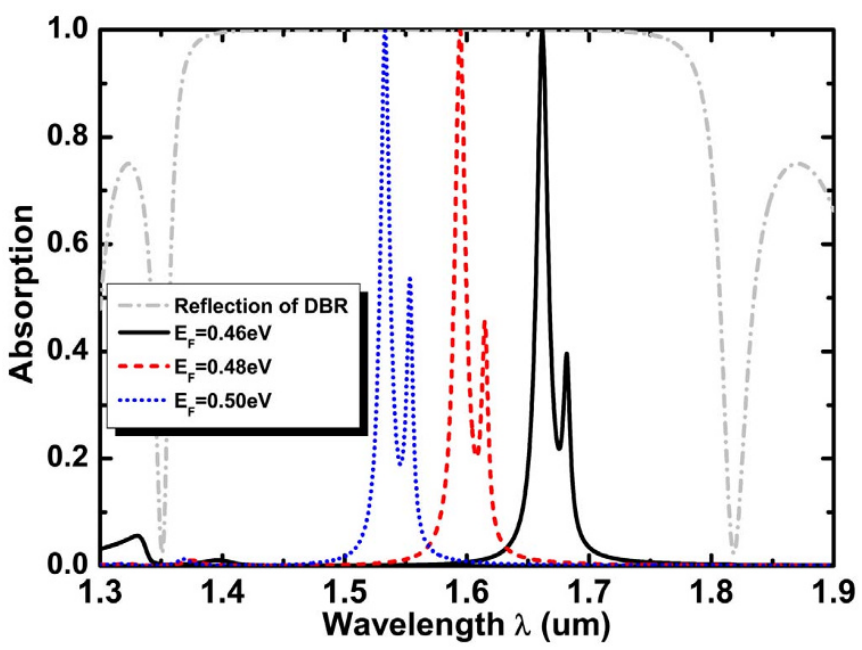

Figure 6 The absorption of critically coupled resonance as a function of work wavelength at different Fermi energy $E_{F}$. Where $t_{s}=590 \mathrm{~nm}$ for $E_{F}$ $=0.50 \mathrm{eV}, \mathrm{t}_{\mathrm{s}}=610 \mathrm{~nm}$ for $\mathrm{E}_{\mathrm{F}}=0.48 \mathrm{eV}$, and $\mathrm{t}_{\mathrm{s}}=650 \mathrm{~nm}$ for $\mathrm{E}_{\mathrm{F}}=$ $0.46 \mathrm{eV}, \mathrm{t}_{\mathrm{HMM}}$ is kept as constant $88 \mathrm{~nm}$. Other parameters are as in Fig. 2. The gray dashed-dotted line gives the reflection of DBR on the glass substrate. 


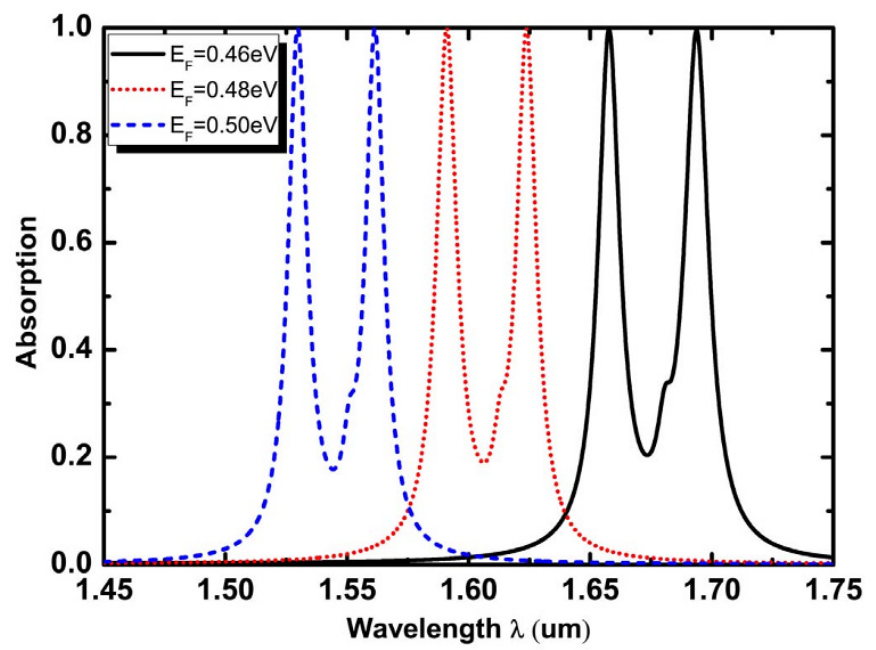

Figure 7 The critical coupling phenomena at two distinct wavelengths at different Fermi energy $E_{\mathrm{F}}$. For $\mathrm{E}_{\mathrm{F}}=0.50 \mathrm{eV}$ we used $\mathrm{t}_{\mathrm{HMM}}=16^{*}\left(\mathrm{t}_{\mathrm{g}}+\mathrm{t}_{\mathrm{d}}\right)$; for $\mathrm{E}_{\mathrm{F}}=0.48 \mathrm{eV}$ we used $\mathrm{t}_{\mathrm{HMM}}=17 *\left(\mathrm{t}_{\mathrm{g}}+\mathrm{t}_{\mathrm{d}}\right)$; for $\mathrm{E}_{\mathrm{F}}=0.46 \mathrm{eV}$ we used $t_{\text {HMM }}=19^{*}\left(t_{\mathrm{g}}+t_{d}\right)$, where $M=1$ and $t_{\mathrm{s}}$ is kept as constant $500 \mathrm{~nm}$. Other parameters are as in Fig. 3.

$650 \mathrm{~nm}$ for $\mathrm{E}_{\mathrm{F}}=0.46 \mathrm{eV}$. It is clear from Fig. 2 that, for $\lambda=$ $1.661 \mathrm{um}$ at $\mathrm{E}_{\mathrm{F}}=0.46 \mathrm{eV}$ departure from $\mathrm{CC}$, one can recover $\mathrm{CC}$ just by adjusting the distance $\mathrm{t}_{\mathrm{s}}=650 \mathrm{~nm}$. For $\lambda=1.594 \mathrm{um}$ at $\mathrm{E}_{\mathrm{F}}=$ $0.48 \mathrm{eV}, \mathrm{CC}$ resonance can be recovered by increasing the distance $\mathrm{t}_{\mathrm{s}}$ to $610 \mathrm{~nm}$. Therefore, our CCR has the potential to achieve tunable $\mathrm{CC}$ frequency in a fixed configuration.

Critical coupling phenomena at two frequencies. From Fig. 3, it is found that there are two absorption peaks in the layered structure. In Fig. 6 we have recovered the CC at the different Fermi energy for one peak. But how can we realize the CC phenomena at two distinct frequencies? By optimizing the parameters of the structure, including $t_{s}$ and $t_{H M M}$, we have plotted the absorption coefficient as a function of wavelength, as shown in Fig. 7. We have used different sets of values for different Fermi energies. For $\mathrm{E}_{\mathrm{F}}=$ $0.50 \mathrm{eV}$ we used $\mathrm{t}_{\mathrm{HMM}}=16^{*}\left(\mathrm{t}_{\mathrm{g}}+\mathrm{t}_{\mathrm{d}}\right)$; for $\mathrm{E}_{\mathrm{F}}=0.48 \mathrm{eV}$ we used $t_{\text {HMM }}=17 *\left(t_{\mathrm{g}}+t_{d}\right)$; for $\mathrm{E}_{\mathrm{F}}=0.46 \mathrm{eV}$ we used $\mathrm{t}_{\mathrm{HMM}}=19 *\left(\mathrm{t}_{\mathrm{g}}+\right.$ $t_{d}$ ), where $M=1$ and $t_{s}=500 \mathrm{~nm}$. It is clear that for optimal values of the thickness of graphene-based HMMs we can obtain CC at two distinct frequencies.

\section{Discussion}

In addition to Fermi energy, the resonant behavior of $\varepsilon_{\mathrm{x}}$ also depends on the fill factions of dielectric and graphene sheet. Decrease in thickness of dielectric $t_{d}$ or increase in layer number of graphene sheets $M$ leads to the decrease of fill factor of dielectric $f_{d}$ and the increase of fill factor of dielectric $f_{g}$, so that the graphene sheets are becoming more and more important in the HMMs. These properties are significant for controlling CC due to the strong dependence of the CC behavior on the resonant behavior of $\varepsilon_{\mathrm{x}}$, while the results are shown in Fig. 8. The solid lines are the CC phenomenon at the central wavelength $\lambda=1.534 \mathrm{um}$ and $\mathrm{E}_{\mathrm{F}}=0.5 \mathrm{eV}$, the parameters are the same as those in Fig. 3. In Fig. 8(a), it is found that the central frequency of the absorption peak shifts towards longer wavelength side with increasing the layer number of the graphene sheets $M$. The absorption peak departs from the CC phenomenon, which may be optimized by varying $t_{H M M}$ or $t_{s}$ as shown in Fig. $S 1$ of the Supplementary materials. In Fig. 8(b), we find that the central frequency of the absorption peak shifts to longer wavelength side with decreasing $t_{d}$ and shifts to shorter wavelength side with increasing $t_{d}$, the optimization result is given in Fig. S2 of the Supplementary

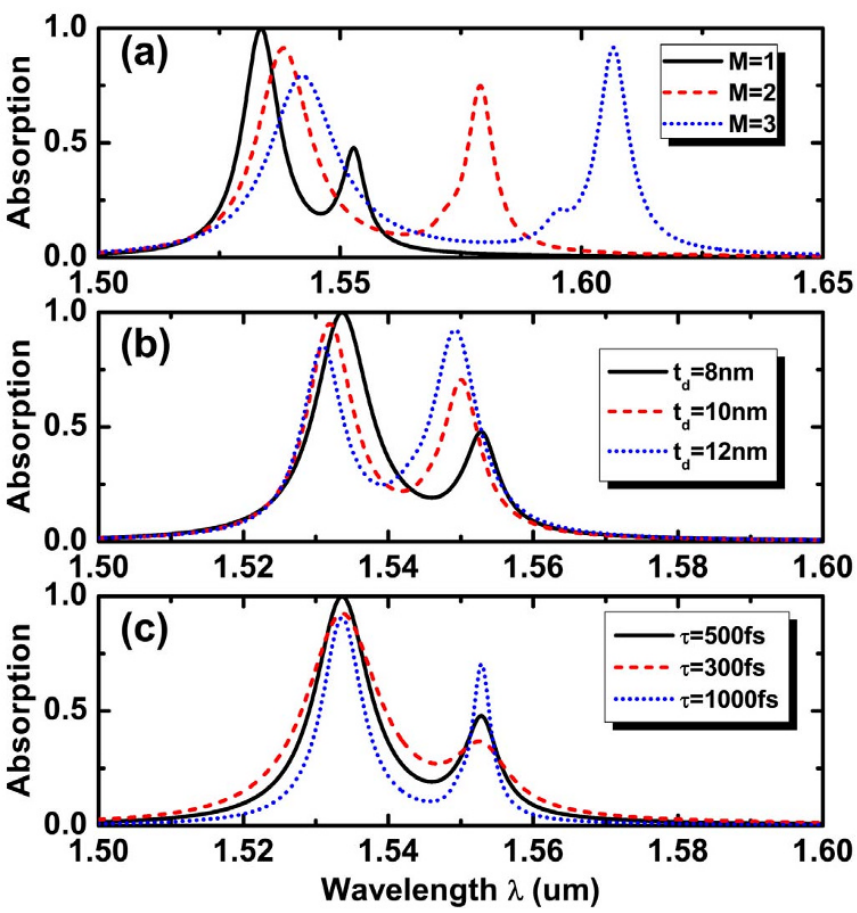

Figure 8 The absorption as a function of work wavelength at the different layer number of graphene sheets (a), thickness of dielectric in graphene-based HMM (b) and relaxing time (c). Where $t_{d}=8 \mathrm{~nm}$ and $\tau$ $=500 \mathrm{fs}$ in (a), $\mathrm{M}=1$ and $\tau=500 \mathrm{fs}$ in (b), and $\mathrm{t}_{\mathrm{d}}=8 \mathrm{~nm}$ and $\mathrm{M}=1 \mathrm{in}$ (c), other parameters are as in Fig. 3.

materials. In Fig. 8(c), we show the absorption as a function of wavelength at different relaxing time $\tau$. It is clear that the central frequency of the absorption peak keeps fixed and the absorption decreases with the decreased or increased relaxing time, the optimization result is given in Fig. S3 of the Supplementary materials. According to above analyses, it is demonstrated that the dependence of absorption on the resonant behavior of $\varepsilon_{\mathrm{x}}$ provides more degree of freedom to control the CC phenomenon at the NIR frequencies by using graphene-based HMMs.

In conclusion, we have presented a graphene-based HMM as the absorbing thin film to realize the CC phenomenon in the NIR frequency regime and discussed the controllable properties of this phenomenon in the CCR. It is found that the CC phenomenon depends on the resonant behavior of the effective permittivity of HMMs, and the CC frequency can be effectively tuned by changing the Fermi energy level applied on the graphene sheets. However, near-perfect absorption departs from the CCR if the Fermi energy is significantly changed. Hence, to recover the CC phenomenon optimization on the thickness of dielectric, the layer number of graphene sheets in HMMs, and the relaxation time of graphene sheet need to satisfy certain requirements. The optimization results are also presented. We believe that the controllable CC phenomenon and near-perfect absorption at the NIR frequencies (telecommunication band) could find potential applications in optical communications, optical detectors and photovoltaic.

\section{Methods}

The numerical calculations for the effective permittivities of the graphene-based hyperbolic metamaterials, the theoretical absorption spectrum, and the optimization results of critical coupling phenomena in the critically coupled resonant containing graphene-based hyperbolic metamaterials were generated using Matlab code based on the transfer matrix method. Let a plane wave be injected from vacuum into the critically coupled resonant at normal incidence with $+z$ direction. Generally, the electric and magnetic fields at any two positions $z$ and $z+\Delta z$ in the same layer can be related via a transfer matrix, 


$$
M_{i}(\Delta z, \omega)=\left(\begin{array}{ll}
\cos \left(k_{i z} \Delta z\right) & -j q_{i z} \sin \left(k_{i z} \Delta z\right) \\
-j \frac{1}{q_{i z}} \sin \left(k_{i z} \Delta z\right) & \cos \left(k_{i z} \Delta z\right)
\end{array}\right)
$$

where $i$ denote the different media, $k_{i z}=\sqrt{k_{0}^{2} \varepsilon_{i}-k_{x}^{2}}$ is the components of the wave vector along the $\mathrm{z}$ axis in the isotropic medium. For the TM polarization,

$k_{z}=\sqrt{k_{0}^{2} \varepsilon_{x}-\left(\varepsilon_{x} / \varepsilon_{z}\right) k_{x}^{2}}$ and $q_{z}=k_{z} / k_{0} \varepsilon_{x}$ in the hyperbolic metamaterial, $q_{i z}=k_{i z} / k_{0} \varepsilon_{i}$ in the isotropic medium. The reflection and transmission coefficients can be calculated as

$$
\begin{aligned}
& r=\frac{\left[X_{1,1}+X_{1,2} * p_{L}\right] * p_{1}-\left[X_{2,1}+X_{2,2} * p_{L}\right]}{\left[X_{1,1}+X_{1,2} * p_{L}\right] * p_{1}+\left[X_{2,1}+X_{2,2} * p_{L}\right]} \\
& t=\frac{2 p_{1}}{\left[X_{1,1}+X_{1,2} * p_{L}\right] * p_{1}+\left[X_{2,1}+X_{2,2} * p_{L}\right]}
\end{aligned}
$$

Hence, the absorption can be obtained as,

$$
A=1-|r|^{2}-\left(p_{L} / p_{1}\right)|t|^{2}
$$

where $X_{i j}(i, j=1,2)$ are the matrix elements of $X_{i, j}(\omega)=\prod M_{i}(\Delta z, \omega)$, which represent the total transfer matrix connecting the fields at the incident end and the exit end; $p_{1}=\sqrt{k_{0}^{2} \varepsilon_{1}-k_{x}^{2}} / \varepsilon_{1}, p_{L}=\sqrt{k_{0}^{2} \varepsilon_{L}-k_{x}^{2}} / \varepsilon_{L}, \varepsilon_{1}$ and $\varepsilon_{L}$ are the permittivity of the media before the incident end and the space after the exit end, respectively.

1. Novoselov, K. S. et al. Electric field effect in atomically thin carbon films. Science 306, 666-669 (2004).

2. Neto, A. H. C., Guinea, F., Peres, N. M. R., Novoselov, K. \& Geim, A. K. The electronic properties of graphene. Rev. Mod. Phys. 81, 109-162 (2009).

3. Grigorenko, A. N., Polini, M. \& Novoselov, K. S. Graphene plasmonics. Nat. Photonics 6, 749-758 (2012).

4. Liu, M. et al. A graphene-based broadband optical modulator. Nature 474, 64-67 (2011).

5. Vakil, A. \& Engheta, N. Transformation optics using graphene. Science 332, 1291-1294 (2011)

6. Koppens, F. H. L., Chang, D. E. \& García de Abajo, F. J. Graphene plasmonics: A platform for strong light-matter interactions. Nano Lett. 11, 3370-3377 (2011).

7. Bao, Q. L. \& Loh, K. P. Graphene Photonics, Plasmonics, and Broadband Optoelectronic Devices. ACS Nano 6, 3677-3694 (2012).

8. Zhang, H., Virally, S., Bao, Q. L., Loh, K. P., Massar, S., Godbout, N. \& Kockaert, P. Z-scan measurement of the nonlinear refractive index of graphene. Opt. Lett. 37, 1856-1858 (2012).

9. Thongrattanasiri, S., Koppens, F. H. L. \& García de Abajo, F. J. Complete optical absorption in periodically patterned graphene. Phys. Rev. Lett. 108, 047401 (2012).

10. Ferreira, A., Peres, N. M. R., Ribeiro, R. M. \& Stauber, T. Graphene-based photodetector with two cavities. Phys. Rev. B 85, 115438 (2012).

11. Ferreira, A. \& Peres, N. M. R. Complete light absorption in graphenemetamaterial corrugated structures. Phys. Rev. B 86, 205401 (2012).

12. Nikitin, A. Y., Guinea, F. \& Martin-Moreno, L. Resonant plasmonic effects in periodic graphene antidot arrays. Appl. Phys. Lett. 101, 151119 (2012)

13. Nefedov, I. S., Valaginnopoulos, C. A. \& Melnikov, L. A. Perfect absorption in graphene multilayers. J. Opt. 15, 114003 (2013).
14. Tischler, J. R., Bradley, M. S. \& Bulović, V. Critically coupled resonantors in vertical geometry using a planar mirror and a $5 \mathrm{~nm}$ thick absorbing film. Opt. Lett. 32, 2045-2047 (2006)

15. Tischler, J. R. et al. Solid state cavity QED: strong coupling in organic thin films. Org. Electron. 8, 94-113 (2007).

16. Gupta, S. D. Strong-interaction-mediated critical coupling at two distinct frequencies. Opt. Lett. 32, 1483-1485 (2007).

17. Deb, S., Gupta, S. D., Banerji, J. \& Gupta, S. D. Critical coupling at oblique incidence. J. Opt. A: Pure Appl. Opt. 9, 555-559 (2007).

18. Deb, S. \& Gupta, S. D. Critical coupling in a Fabry-Pérot cavity with metamaterial mirrors. Opt. Commun. 283, 4764-4769 (2010).

19. Reddy, K. N., Gopal, A. V. \& Gupta, S. D. Nonlinearity induced critical coupling. Opt. Lett. 38, 2517-2520 (2013).

20. Iorsh, I. V., Mukhin, I. S., Shadrivov, I. V., Belov, P. A. \& Kivshar, Y. S. Hyperbolic metamaterials based on multilayer graphene structures. Phys. Rev. B 87, 075416 (2013).

21. Othman, M. A. K., Guclu, C. \& Capolino, F. Graphene-based tunable hyperbolic metamaterials and enhanced near-field absorption. Opt. Express 21, 7614-7632 (2013).

22. Sreekanth, K. V., De Luca, A. \& Strangi, G. Negative refraction in graphene based metamaterials. Appl. Phys. Lett. 103, 023107 (2013).

23. Zhang, T., Chen, L. \& Li, X. Graphene-based tunable broadband hyperlens for farfield subdiffraction imaging at mid-infrared frequencies. Opt. Express 21, 20888-20899 (2013).

24. Chen, P. Y. \& Alù, A. Atomically thin surface cloak using graphene monolayers. ACS Nano 5, 5855-5863 (2011)

25. Hanson, G. W. Dyadic Green's functions and guided surface waves for a surface conductivity model of graphene. J. Appl. Phys. 103, 064302 (2008).

26. Gusynin, V. P., Sharapov, S. G. \& Carbotte, J. P. Magneto-optical conductivity in graphene. J. Phys.: Condens. Matter 19, 026222 (2007).

\section{Acknowledgments}

This work is partially supported by the Minister of Education (MOE) Singapore under the grant no. 35/12, Natural Science Foundation of Hunan Province of China (Grant No. 12JJ7005), the National Natural Science Foundation of China (Grant No. 11004053), and the Ph.D. Programs Foundation of Ministry of Education of China (Grant No. 20120161120013)

\section{Author contributions}

Y.J.X. and X.Y.D. performed derivation of formulas and wrote the manuscript. J.G. helped in numerical calculations. H.Z., S.C.W. and D.Y.T. conceived the idea and co-wrote the manuscript. All authors discussed the results and commented on the manuscript.

\section{Additional information}

Supplementary information accompanies this paper at http://www.nature.com/ scientificreports

Competing financial interests: The authors declare no competing financial interests. How to cite this article: Xiang, Y. et al. Critical coupling with graphene-based hyperbolic metamaterials. Sci. Rep. 4, 5483; DOI:10.1038/srep05483 (2014).

This work is licensed under a Creative Commons Attribution-NonCommercialNoDerivs 4.0 International License. The images or other third party material in this article are included in the article's Creative Commons license, unless indicated otherwise in the credit line; if the material is not included under the Creative Commons license, users will need to obtain permission from the license holder in order to reproduce the material. To view a copy of this license, visit http:// creativecommons.org/licenses/by-nc-nd/4.0/ 